\title{
ANÁLISE NUMÉRICO-EXPERIMENTAL DA AGITAÇÃO DE CALDA EM RESERVATÓRIOS DE PULVERIZADORES AGRÍCOLAS
}

Doi:http://dx.doi.org/10.1590/1809-4430-Eng.Agric.v35n6p 1065-1078/2015

\section{GUSTAVO B. MICHELI ${ }^{1}$, ALCIDES PADILHA ${ }^{2}$, VICENTE L. SCALON ${ }^{3}$}

\begin{abstract}
RESUMO: Neste trabalho apresenta-se um estudo numérico-experimental do comportamento da agitação de fluido em reservatórios de pulverizadores agrícolas. Uma adequada agitação da calda de defensivos agrícolas em um reservatório é muito importante para garantir a ho mogeneidade da pulverização ao longo de toda a cultura. O objetivo do estudo foi avaliar a viabilidade da utilização de um modelo computacional aplicado ao problema de agitação de calda em reservatórios de pulverizadores agrícolas, e assim, auxiliar no projeto dos mesmos, com economia de tempo e custo. Deste modo, resultados de simulações computacionais de fluidodinâmica (CFD) foram comparados a testes experimentais normalizados. Utilizando um modelo monofásico (água), avaliaram-se os perfis de velocidades e de tensões de cisalhamento na parede do reservatório, a fim de possibilitar a visualização do comportamento do fluido em agitação e relacionar essas variáveis com a concentração de resíduos. A comparação entre resultados experimentais e o modelo computacional desenvolvido mostrou-se viável, indicando que regiões com baixos valores numéricos de tensão de cisalhamento estavam em concordância com as regiões de alta deposição de cobre da solução-teste.
\end{abstract}

PALAVRAS-CHAVE: agitação em reservatórios, pulverização agrícola, fluidodinâmica computacional.

\section{NUMERICAL AND EXPERIMENTAL ANALYSIS OF PESTICIDE SPRAY MIXING IN SPRA YER TANKS}

\begin{abstract}
This paper presents a numerical and experimental study of spray fluid performance with stirring in tanks of agricultural sprayers. The pesticide spray should be stirred properly to ensure spraying homogeneity throughout the entire crop. The aim of this study was to evaluate the feasibility of using computer models in pesticide spray stirring and, thus, reducing operational time and costs. Therefore, results of computer simulations of fluid dynamics (CFD) were compared to standard experimental tests. We evaluated shear stress and speed profiles against tank wall using a monophasic model (water). Thus, we could verify the fluid performance during stirring, and relate these variables with residue concentrations. The comparison between experimental and computational model was feasible since areas with lower numerical values of shear stress were in line with areas with high copper deposition.
\end{abstract}

KEYWORDS : tank stirring, farm spraying, computational fluid dynamics.

\section{INTRODUÇÃO}

A eficiência da homogeneização das caldas de defensivos agrícolas ao longo do reservatório de um pulverizador tem grande impacto na qualidade da aplicação e no tratamento de uma cultura. Os solventes utilizados, normalmente água, são substâncias usadas para reduzir a concentração do princípio ativo na formulação. Podem ajudar na ação mecânica do produto, mas não influir diretamente em sua toxidez, sendo, portanto, inertes ao agente de dano. A subdosagem ou superdosagem de um determinado agroquímico no tratamento de uma cultura pode causar-lhe danos, tornando muito importante a homogeneização dessa solução.

\footnotetext{
${ }^{1}$ Eng $^{\mathrm{o}}$ Mecânico, Doutorando em En genharia Mecânica, Programa de Pós-Graduação em En genharia Mecânica, Unesp/Bauru - SP, Fone: (14) 98131-6611, gustavobm.micheli@gmail.com

${ }^{2}$ Eng $^{\circ}$ Mecânico, Prof. Doutor, Departamento de Engenharia Mecânica, Unesp/Bauru - SP, padilha@ feb.unesp.br

${ }^{3}$ Eng $^{\circ}$ Mecânico, Prof. Doutor, Departamento de Engenharia Mecânica, Unesp/Bauru - SP, scalon@ @eb.unesp.br 
UCAR et al. (2000) investigaram a eficácia do sistema de agitação hid ráulica em reservatórios de pulverizadores agrícolas, medindo a concentração de uma solução de água e cal, que sai do reservatório e é pulverizada atra vés dos bicos na barra de pulverização. Foi reforçada a importânc ia das tecnologias necessárias para um pulverizador agrícola para minimizar os problemas ambientais associados com os pesticidas e ao mesmo tempo alcançar o controle efetivo e econômico de doenças. Sem uma agitação eficiente nos reservatórios, a maioria dos pós molháveis e de outras formulações sólidas de pesticidas possui a tendência de permanecer depositada nas paredes dos reservatórios, principalmente nos vincos da geometria e no fundo do mesmo.

Complementando a este estudo, UCAR et al. (2001) utilizaram o código comercial de fluidodinâmica FLUENT para investigar o comportamento dos fluidos em reservatórios de pulverizadores e fazer comparações com os resultados experimentais previamente obtidos. Foram feitas comparações quantitativas entre 12 simulações monofásicas e resultados experimentais de velocidades medidas em 9 posições dentro de um reservatório com capacidade de $1.136 \mathrm{~L}$, através de anemômetros de fio quente. Em 118 de 144 velocidades medidas, a diferença em relação às velocidades simuladas computacionalmente foi de aproximadamente $50 \%$. Todas as medidas foram feitas no plano de um dos agitadores, e os anemômetros foram colocados alinhados ao eixo longitudinal do reservatório. Não foi possível obter des vios menores devido à turbulência gerada na agitação, pois as respostas dos anemômetros são dos fluxos na mesma direção em que foram alinhados. Os valores de velocidades simuladas tenderam a serem maiores que os medidos nas regiões próximas ao topo do reservatório, e sempre menores na região central do mesmo.

SPOGIS (2002) fez estudos de agitação em reservatórios de misturas utilizados na indústria química, petroquímica, farmacêutica e alimentícia, com diferentes modelos de agitadores mecânicos, comparando o bombeamento e os padrões de circulação gerados no reservatório, além de curvas características de cada agitador, atra vés da fluidodinâmica computacional. Os resultados desse trabalho mostraram que agitadores com formato de âncora (que geram fluxos tangenciais) apresentam bom desempenho em regime laminar. Os demais tipos apresentam melhor desempenho em regime turbulento, pois em regime laminar, o fluxo gerado por estes agitadores não é bem distribuído por todo o reservatório devido à baixa dissipação de energia e de turbulência.

Há também muitos exemplos de estudos com simulação numérica de comportamentos de fluidos nas áreas médica e biomecânica. MAES et al. (2012), estudaram a utilização da CFD na área de reconstituição óssea in vitro, com a utilização de estímulos mecânicos gerados por movimentação de fluidos através da matriz óssea. O intuito é minimizar o problema de não uniformidade da seção óssea, que pode ocorrer nos procedimentos utilizados atualmente, tais como o enxerto. Nesse estudo foi utilizada a avaliação da tensão de cisalhamento na parede celular para estimar, quantitativamente, o ambiente fluidodinâmico na matriz.

Esses estudos permitiram contextualizar o problema da agitação de fluido em reservatórios e auxiliar o presente trabalho com alternativas de metodologias que têm sido utilizadas para a solução do problema.

Além disso, os custos e o tempo de desenvolvimento em projetos de reservatórios para pulverizadores agrícolas são altos, pois normalmente as empresas de máquinas agrícolas acabam investindo na construção de moldes para processos como rotomoldagem, RTM/RTMlight, sopro e injeção de plásticos. Tem-se o objetivo de não gerar novos custos com possíveis retrabalhos nas ferramentas devido a erros de projeto. Em alguns casos, as alterações necessárias para garantir a boa agitação do reservatório e a não deposição de produtos com alta concentração nas paredes do mesmo podem ser inviáveis e corresponder até à perda da ferramenta de fabricação, gerando custos ainda maiores.

No intuito de reduzir os custos com protótipos dos reservatórios, pode-se utilizar a simulação numérica dos fenômenos envolvidos na agitação, sendo obtida com o auxílio de um código de fluidodinâmica computacional (CFD), que, em uma análise tridimensional, utiliza a técnica de volumes finitos como método de discretização dos domínios fluidos. 
A fluidodinâmica computacional é uma técnica numérica de resolução das equações que governam o escoamento de fluidos em uma determinada geometria: as equações de conservação de massa, momento e energia (Equações de Transporte de Navier-Stokes) e as equações de estado (AUGUSTO et al., 2010).

O objetivo deste estudo foi avaliar a deposição de particulado sólido e a agitação de calda dos reservatórios dos pulverizadores agrícolas através da simulação numérica de CFD, visualizando o comportamento do fluido no modelo numérico e realizando a comparação com os resultados experimentais obtidos.

\section{MATERIAL E MÉTODOS}

Os testes de validação de um reservatório e o sistema de agitação do mesmo para um pulverizador agrícola são normalizados segundo norma ISO 5682-2 (ISO, 1997). Para essa avaliação, utiliza-se de um reservatório de plástico rotomoldado com capacidade de 4000 litros, instalado em um turbopulverizador agrícola para culturas de frutas cítricas. O sistema de agitação é composto por dois agitadores, um hidráulico e outro mecânico (tipo hélice). Prepara-se uma solução de $1 \%$ de oxiclore to de cobre no reservatório e avalia-se a eficiência de agitação após 10 minutos de funcionamento do agitador (em todas as amostragens). A avaliação é realizada em três níveis do reservatório. A Figura 1 mostra como foram determinadas as regiões de coleta das amostras para a verificação da concentração de cobre ao longo dos três níveis do reservatório.

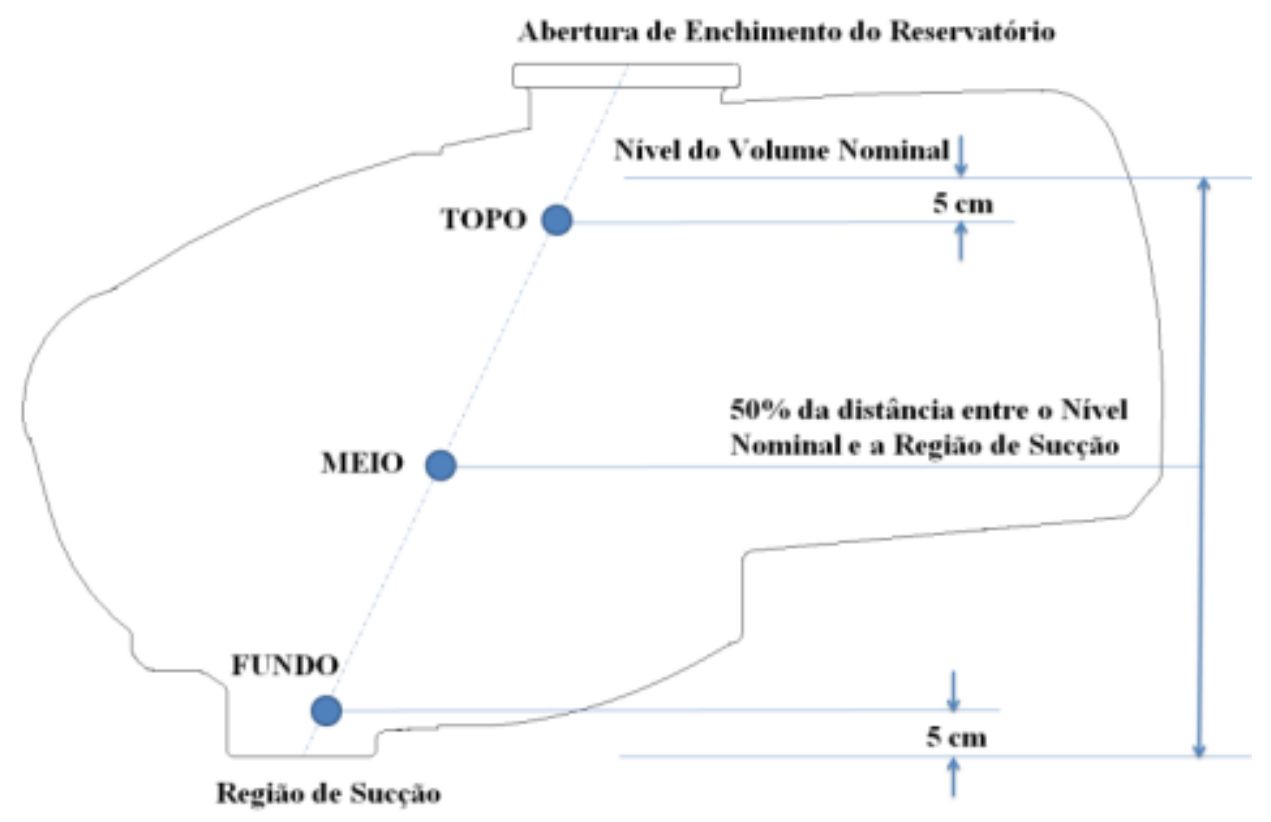

FIGURA 1. Posições de coleta das amostras. Sampling spots.

Avaliou-se a eficiência durante a agitação inicial (1 $1^{\underline{a}}$ amostragem), na reagitação após 16 horas de repouso ( $2^{\mathrm{a}}$ amostragem) e durante o processo de esvaziamento do reservatório. Foram coletadas 3 amostras de cada nível do reservatório, e a análise foi realizada com os valores médios.

O desvio máximo permitido pela norma prEN 12761-2 (ENTAM, 1997) na 1a amostragem ou concentração-base é de 0,95 a $1,05 \%$. Para a $2^{-}$amostragem e durante o teste de esvaziamento do reservatório, o desvio máximo permitido em relação à concentração-base é de $\pm 15 \%$.

O processo de esvaziamento ocorreu com o auxílio de uma bomba de pulverização e uma válvula de descarga após a bomba. As amostras durante esse processo foram coletadas a cada retirada de $200 \mathrm{~L}$ da solução do reservatório. A primeira amostra do esvaziamento foi coletada 5 segundos após o primeiro fluxo de solução-teste sair pela válvula de descarga, e a última amostra, no momento em que a bomba teve a primeira sucção de ar. 
A determinação da concentração de cobre foi feita através do método de diferença de massa, em que uma amostra da solução, cuja massa é conhecida, é submetida a um processo de secagem em estufa ventilada, com temperatura entre 105 e $110^{\circ} \mathrm{C}$.

\section{Modelamento numé rico}

As simulações numéricas foram realizadas utilizando o código de CFD comercial, ANSYS CFX 14.5. Neste trabalho, foi utilizado um modelo monofásico (água), para avaliar os perfis de velocidades e de tensões de cisalhamento na parede do reservatório e verificar as regiões de baixo fluxo como potenciais concentradores de resíduos. Em simulações de fluxos multifásicos sólido-líquido, a solução que permite a visualização de um mapa de concentração do sólido, na solução aquosa, no interior do reservatório, é o modelo Euler-Euler (TAMBURINI et al., 2011). Porém, essa solução requer um esforço computacional muito maior que uma solução com um modelo monofásico, e a concentração do sólido em questão (oxicloreto de cobre) é de apenas 1\%, permitindo essa simplificação.

Dessa forma, foi possível verificar a viabilidade de relacionar a velocidade do fluido e a tensão de cisalhamento na parede do reservatório com a concentração de cobre encontrada nos testes experimentais e identificar as regiões de alta deposição de cobre da solução-teste.

\section{Equações de conservação}

As equações que governam a solução numérica do problema são as equações de: conservação da massa, [eq. (1)]; transporte da quantidade de movimento, [eq. (2)]; e conservação da energia, [eq. (3)]. Como se trata de um escoamento com regiões turbulentas, para determinar a viscosidade turbulenta $(\mu \mathrm{t})$, é preciso determinar os termos adicionais que foram resolvidos atra vés da utilização do modelo de turbulência de duas equações k-epsilon, eqs. (4) e (5):

$$
\begin{aligned}
& \frac{\partial \rho}{\partial \mathrm{t}}+\frac{\partial}{\partial \mathrm{x}_{\mathrm{j}}}\left(\rho \mathrm{U}_{\mathrm{j}}\right)=0 \\
& \frac{\partial}{\partial \mathrm{t}}\left(\rho \mathrm{U}_{\mathrm{i}}\right)+\frac{\partial}{\partial \mathrm{x}_{\mathrm{j}}}\left(\rho \mathrm{U}_{\mathrm{i}} \mathrm{U}_{\mathrm{j}}\right)=-\frac{\partial \mathrm{P}}{\partial \mathrm{x}_{\mathrm{i}}}-\frac{\partial \mathrm{r}_{\mathrm{j}}}{\partial \mathrm{x}_{\mathrm{j}}}+\rho \mathrm{f}_{\mathrm{i}} \\
& \frac{\partial}{\partial \mathrm{t}}\left(\frac{1}{2} \rho \mathrm{u}^{2}\right)=-\frac{\partial}{\partial \mathrm{x}_{\mathrm{i}}}\left(\frac{1}{2} \rho \mathrm{u}^{2} \mathrm{U}_{\mathrm{i}}\right)-\frac{\partial}{\partial \mathrm{x}_{\mathrm{i}}}\left(\mathrm{PU}_{\mathrm{i}}\right)-\frac{\partial}{\partial \mathrm{x}_{\mathrm{i}}}\left(\mathrm{U}_{\mathrm{i}} \mathrm{\tau}_{\mathrm{ij}}\right)+\rho\left(\mathrm{U}_{\mathrm{i}} \mathrm{f}_{\mathrm{i}}\right) \\
& \frac{\partial}{\partial \mathrm{t}}(\rho \mathrm{k})+\frac{\partial}{\partial \mathrm{x}_{\mathrm{j}}}\left(\rho \mathrm{U}_{\mathrm{j}} \mathrm{k}\right)=\frac{\partial}{\partial \mathrm{x}_{\mathrm{j}}}\left[\left(\mu+\frac{\mu_{\mathrm{t}}}{\sigma_{\mathrm{k}}}\right) \frac{\partial \mathrm{k}}{\partial \mathrm{x}_{\mathrm{j}}}\right]+\mathrm{P}_{\mathrm{k}}-\rho \varepsilon+\mathrm{P}_{\mathrm{kb}} \\
& \frac{\partial}{\partial \mathrm{t}}(\rho \varepsilon)+\frac{\partial}{\partial \mathrm{x}_{\mathrm{j}}}\left(\rho \mathrm{U}_{\mathrm{j}} \varepsilon\right)=\frac{\partial}{\partial \mathrm{x}_{\mathrm{j}}}\left[\left(\mu+\frac{\mu_{\mathrm{t}}}{\sigma_{\varepsilon}}\right) \frac{\partial \varepsilon}{\partial \mathrm{x}_{\mathrm{j}}}\right]+\frac{\varepsilon}{\mathrm{k}\left(\mathrm{C}_{\varepsilon 1} \mathrm{P}_{\mathrm{k}}-\mathrm{C}_{\varepsilon 2} \rho \varepsilon+\mathrm{C}_{\varepsilon 1} \mathrm{P}_{\varepsilon b}\right)}
\end{aligned}
$$

em que,

$\rho$ é a massa específica do fluido;

té o tempo;

$\mathrm{U}_{\mathrm{j}}$ é o vetor velocidade do escoamento;

$\mathrm{P}$ é a pressão estática;

$\tau_{\mathrm{ij}}$ é o tensor viscoso;

$\mathrm{f}_{\mathrm{i}}$ são as forças de campo;

$u$ é a velocidade escalar na direção do escoamento;

$\mu$ é a viscosidade dinâmica do fluido;

k é a energia cinética turbulenta; 
$\varepsilon$ é a taxa de dissipação da energia cinética turbulenta;

$\mathrm{P}_{\mathrm{kb}}$ e $\mathrm{P}_{\varepsilon b}$ representam a influência das forças de corpo;

$\mathrm{P}_{\mathrm{k}}$ é a produção de turbulência devido às forças viscosas,

$\mathrm{C}_{\varepsilon 1}, \mathrm{C}_{\varepsilon 2}, \sigma_{\mathrm{k}}$ e $\sigma_{\varepsilon}$ são constantes empíricas com os valores de 1,$44 ; 1,92 ; 1,0$ e 1,3, respectivamente (ANSYS, 2012).

\section{Caracterizações da utilização da CFD}

A utilização da simulação em CFD passa por 4 etapas principais: geração de malha a partir de um modelo 3D, pré-processamento (definição das condições de contorno), solução iterativa e pós- processamento.

Os modelos 3D do reservatório de 4.000 litros e dos agitadores são mostrados na Fig. 2. Na geração de malha, foram criados 3 domínios diferentes, sendo 2 domínios estacionários (o reservatório e o agitador hidráulico) e 1 domínio rotativo (agitador mecânico). Dessa forma foi possível otimizar o número de elementos do modelo, refinando a malha apenas nas regiões de interesse (próximo à parede do reservatório, às pás do agitador mecânico e às paredes do agitador hidráulico). As malhas dos domínios foram feitas através do código comercial ANSYS ICEM CFD 5.0, sendo definidas após um estudo de convergência de malha e podem ser visualizadas na Fig. 3. O número de elementos da malha do reservatório, do agitador mecânico e do agitador hidráulico foram, respectivamente, de 1.708.210, 130.464 e 263.909 elementos.

No pré-processamento, a definição dos parâmetros do modelo é descrita brevemente como segue: fluido definido como água, com temperatura de referência igual a $25^{\circ} \mathrm{C}$; simulação em regime permanente; escoamento isotérmico; modelo de turbulência K-Epsilon; rotação do agitador mecânico igual a 540 rpm; vazão de entrada pelo agitador hidráulico igual a 4,667 kg/s; vazão de saída pelo agitador hidráulico igual a $3,667 \mathrm{~kg} / \mathrm{s}$, e vazão de saída do fundo do reservatório igual a $1 \mathrm{~kg} / \mathrm{s}$ (esses valores de vazão são característicos do sistema de agitação hidráulica do pulverizador). A convergência numérica adotada ocorreu no momento em que a soma dos resíduos normalizados fosse menor que 1E-04 para todas as variáveis. A simulação foi realizada numa CPU com processador Intel(R) Xeon(R) E5-1660 - 3,3 GHz, com 16 GB de memória RAM e sistema operacional Windows 7. O critério de convergência primário foi a estabilização do torque do agitador mecânico. A simulação teve duração de uma hora e vinte e dois minutos até à convergência.

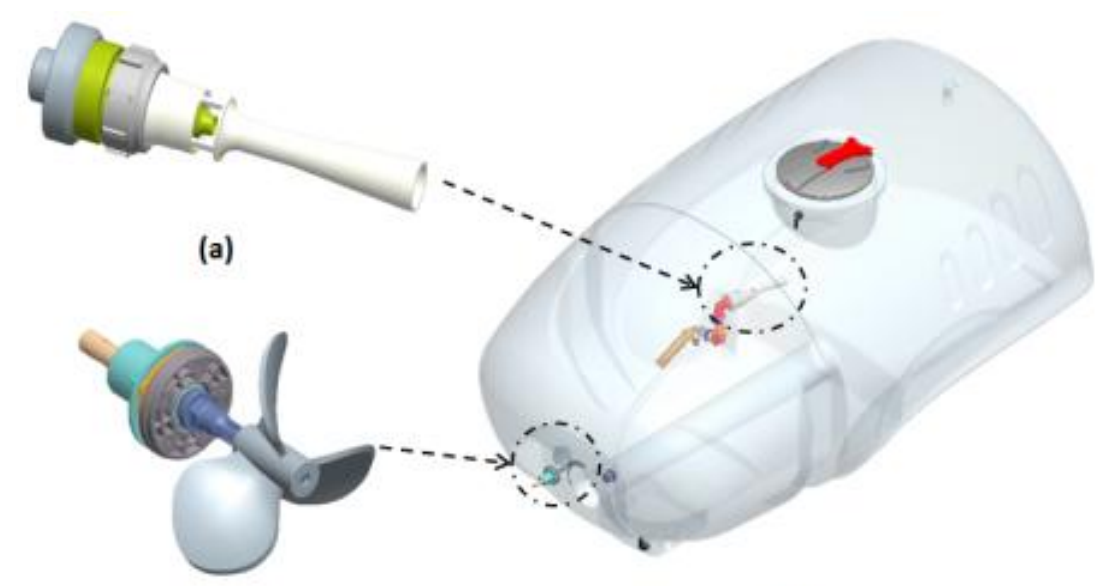

(b)

(c)

FIGURA 2. Modelos 3D: (a) agitador hidráulico; (b) agitador mecânico; e (c) montagem completa do reservatório de 4.000 L. Three-dimensional models: (a) hydraulic mixer, (b) mechanical mixer, and (c) 4000-L tank complete assembling. 


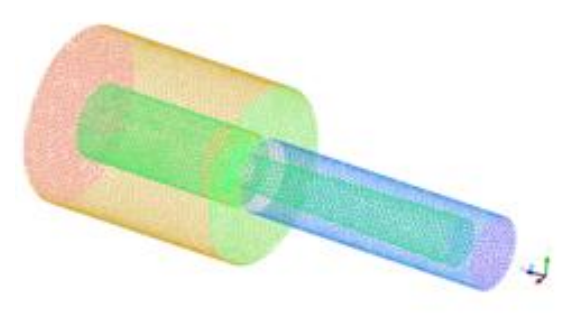

(a)

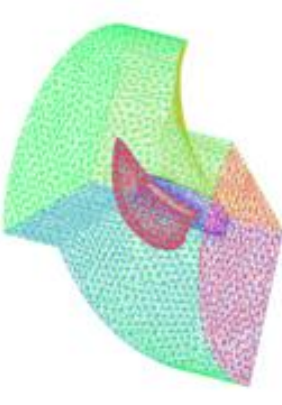

(b)

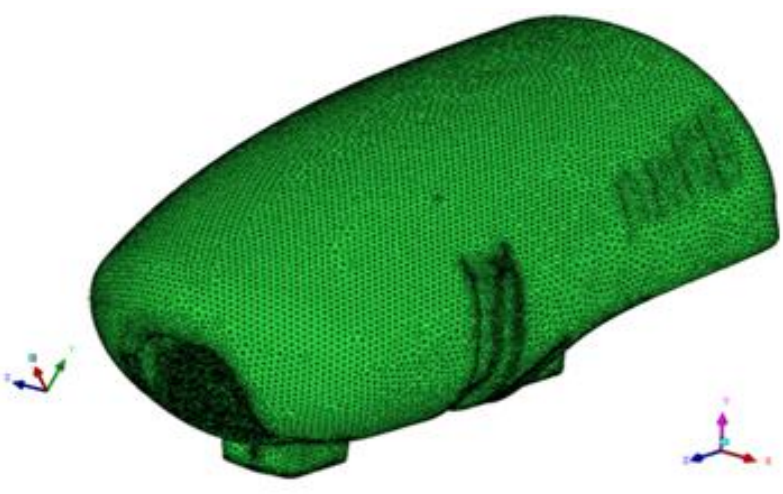

(c)

FIGURA 3. Malhas: (a) agitador hidráulico; (b) agitador mecânico; e (c) reservató rio. Meshes: (a) hydraulic mixer, (b) mechanical mixer, and (c) tank.

\section{RESULTADOS E DISCUSSÃO}

Com os resultados obtidos neste estudo numérico-experimental, foi possível avaliar a viabilidade de utilização dos perfis de velocidades obtidos pela simulação numérica para a previsão da concentração, nas regiões de interesse do volume do reservatório, e dos perfis de tensões de cisalhamento, na parede do mesmo, para prever as regiões de deposição de cobre.

\section{Concentração de oxicloreto de cobre no interior do Reservatório}

\section{Resultados Experimentais}

Neste estudo, foi utilizada uma concentração de oxicloreto de cobre de $1,1 \%$, sendo uma condição de teste um pouco mais severa que a indicada pela norma ISO 5682-2 (ISO, 1997). Não há problemas com esse procedimento, já que o objetivo deste estudo foi avaliar apenas a simulação numérica, como forma de prever os resultados encontrados experimentalmente. Na Fig.4, é possível visualizar os valores médios da concentração de cobre nos testes experimentais, durante a primeira amostragem e a segunda (após 16 horas de repouso, seguidos de 10 minutos de agitação).

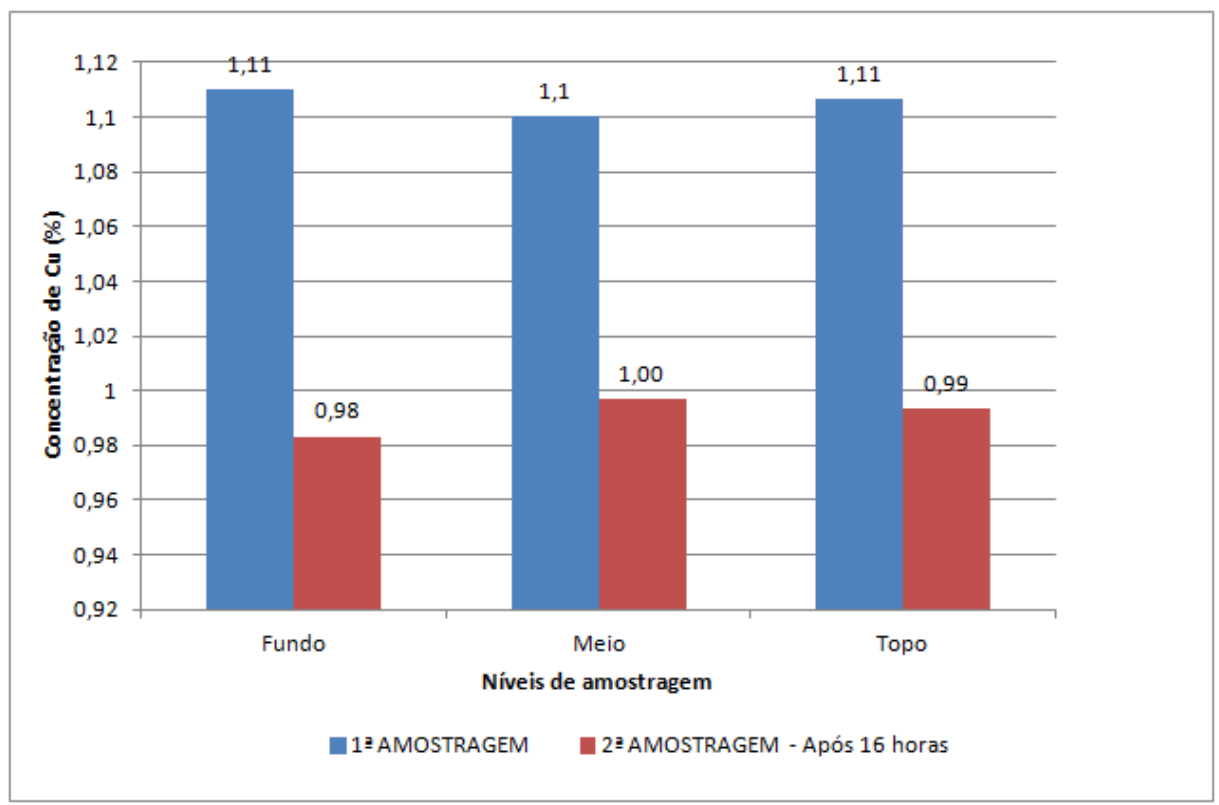

FIGURA 4. Concentração de cobre nos níveis de amostragem do reservatório. Sampling levels of copper concentration in the tank.

Analisando a Fig. 4, é possível perceber que, em ambas as coletas, a variação entre os níveis do reservatório foi inferior a $2 \%$. Porém, para a segunda coleta, os valores médios da concentração 
de cobre nas regiões indicadas ficaram abaixo da faixa normalizada (+/- 5\% da concentração-base). Esse fato é resultado da deposição de cobre na parte inferior da parede do reservatório, ocorrida após as 16 horas de repouso, diminuindo a concentração no interior do mesmo.

\section{Análise dos perfis de velocidades no escoamento}

Como resultado da simulação numérica, é possível visualizar os perfis de velocidades no interior do reservatório, para caracterizar o escoamento durante a agitação e tentar encontrar uma relação entre os valores de velocidade nos níveis do reservatório e as concentrações de cobre obtidas experimentalmente.

A Fig.5 mostra as posições dos planos de corte para a análise dos perfis de velocidade durante a agitação no reservatório. Os perfis de velocidade são mostrados na Fig. 6.

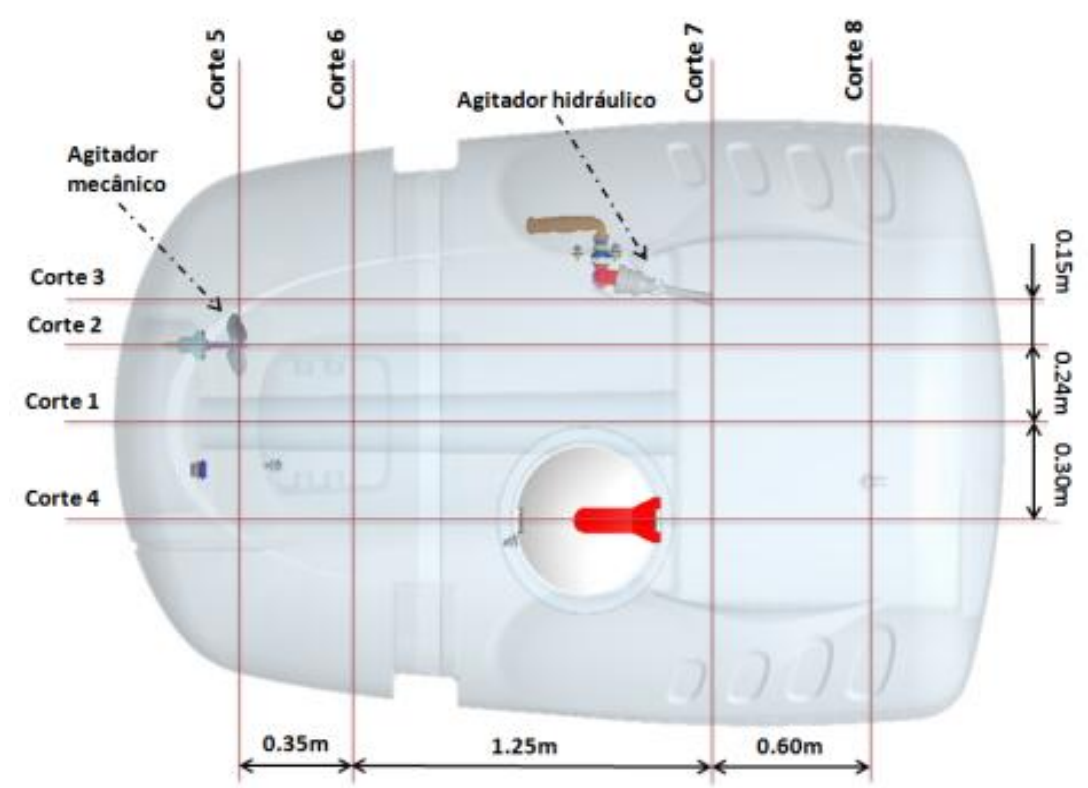

FIGURA 5. Posição dos planos de corte no reservatório. Cutting plan spots within the tank.

A Tab. 1 mostra os valores de concentração média de cobre (evidenciados na Fig.4) e os valores das velocidades médias simuladas a partir de cinco pontos escolhidos próximos às posições das coletas experimentais, delimitando cada nível de amostragem. A velocidade média do fluido é maior no fundo do reservatório que nas demais regiões, devido a esta região estar mais próxima ao agitador mecânico. As três regiões apresentam valores muito próximos de concentração (diferença inferior a 2\%) e valores muito diferentes de velocidades (o valor da velocidade média no fundo é aproximadamente 3 vezes maior que o valor da velocidade média no meio do reservatório). Não foi possível identificar nenhuma tendência ou relação direta entre os valores médios da concentração medida experimentalmente $\mathrm{e}$ as velocidades simuladas.

Outra forma de avaliar o comportamento da agitação é a utilização de uma isossuperfície (representação através de uma superfície tridimensional de regiões com mesmo módulo de uma determinada variável), mostrada na Fig. 7. Nessa representação, a superfície indicada corresponde a todos os elementos que possuem como velocidade média resultante o valor definido pelo usuário.

As Fig. 6 e 7 mostram a influência dos agitadores (hidráulico e mecânico) nos perfis de velocidade ao longo do volume do reservatório. É possível notar que o agitador hidráulico, posicionado conforme Fig. 5, contribuiu parcialmente com a agitação da calda na região plana na parte inferior do reservatório (perfis de velocidade dos planos de corte 2 e 3 ). Existe uma região de sombra de agitação, que não é atingida pelo agitador hidráulico (perfis de velocidade dos planos de corte 1 e 4). Isso também pode ser visualizado na isossuperfície da Fig. 7, onde as velocidades nas regiões fora da isossuperfície são inferiores a $0,3 \mathrm{~m} / \mathrm{s}$. Já o agitador mecânico possui maior influência sobre a agitação do reservatório, garantindo maior gradiente de velocidade em toda a região, no fundo do mesmo. Porém, ele não é capaz de contribuir da mesma forma na direção 
longitudinal do reservatório, devido à sua característica construtiva e à posição de instalação do mesmo na montagem completa.

A Fig. 8 mostra uma representação vetorial do fluido em agitação devido à influência do agitador mecânico no plano de corte 2, da Fig. 6. É possível identificar regiões de sucção do agitador, com velocidades inferiores a $1 \mathrm{~m} / \mathrm{s}$ (regiões radial e traseira da hélice), e de recalque, com velocidades superiores a $1 \mathrm{~m} / \mathrm{s}$ (região frontal da hélice).

TABELA 1. Valores das concentrações médias de $\mathrm{Cu}$ experimentais e das velocidades simuladas. Experime ntal copper average concentrations and simulated speeds.

\begin{tabular}{l|ll|llllll}
\hline \multirow{2}{*}{ Níveis de amostragem } & \multicolumn{6}{l}{ Concentração média de $\mathrm{Cu}(\%)$} & \multicolumn{5}{l}{ Velocidades simuladas (m/s) } \\
\cline { 2 - 9 } & $1^{\mathrm{a}}$ a mostragem & $2^{\mathrm{a}}$ a mostragem & Ponto 1 & Ponto 2 & Ponto 3 & Ponto 4 & Ponto 5 & Média \\
\hline Fundo & 1,11 & 0,98 & 0,49 & 0,54 & 0,5 & 0,51 & 0,55 & 0,52 \\
Meio & 1,10 & 1,00 & 0,16 & 0,17 & 0,16 & 0,20 & 0,19 & 0,18 \\
Topo & 1,11 & 0,99 & 0,28 & 0,25 & 0,24 & 0,24 & 0,26 & 0,25 \\
\hline
\end{tabular}
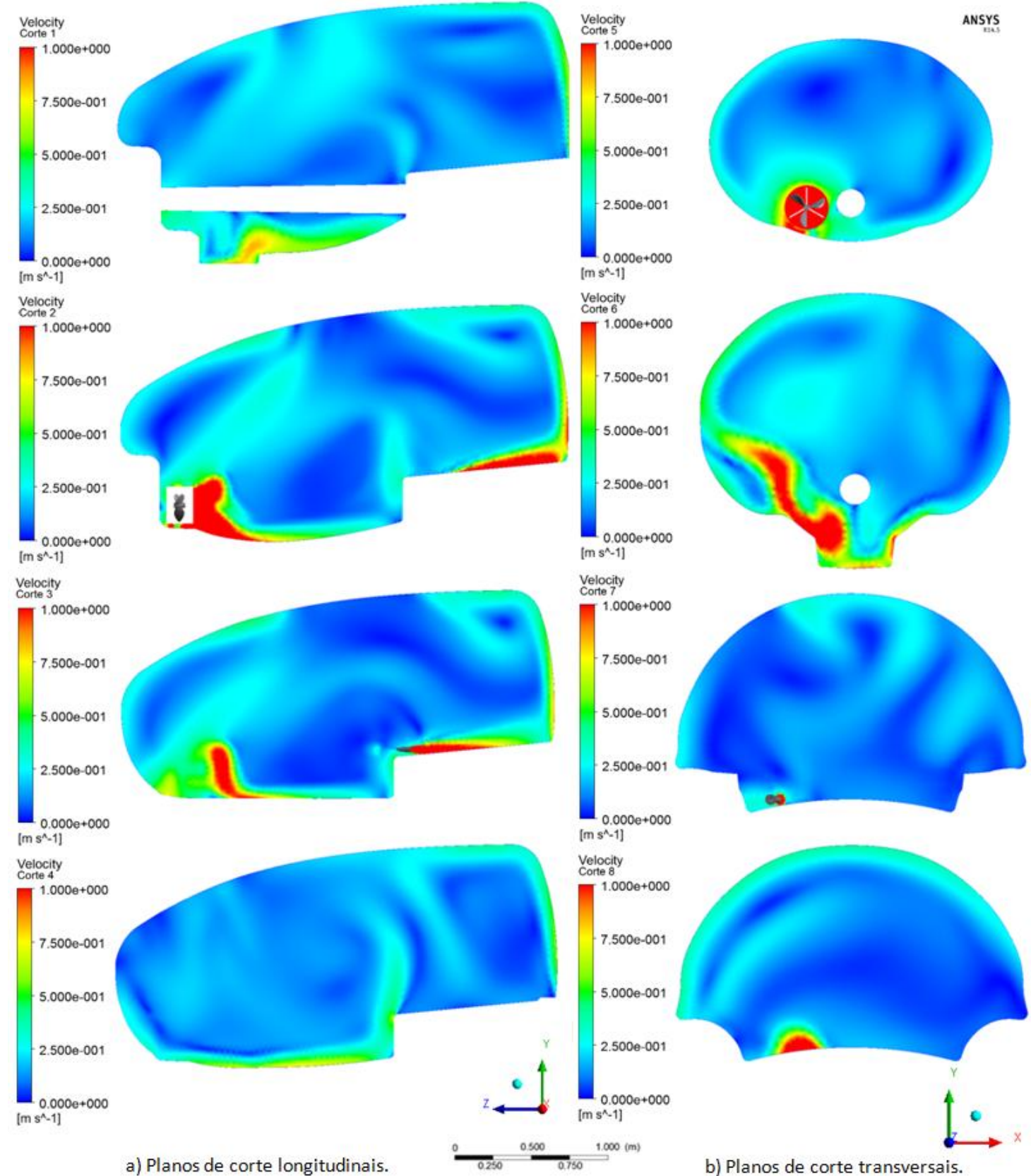

Planos de corte transversais.

FIGURA 6. Vistas dos perfis de velocidade nos planos de corte longitudinais. Speed profile views along the longitudinal cutting plans. 


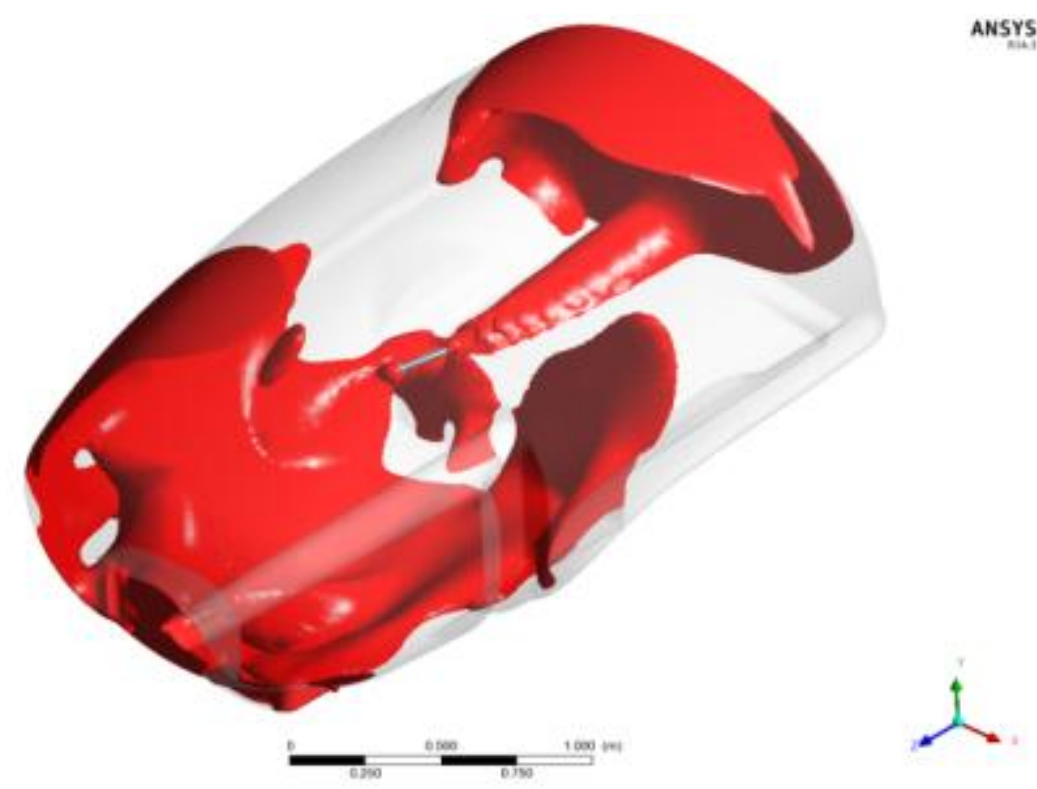

FIGURA 7. Isossuperfície com módulo de velocidade igual a $0,3 \mathrm{~m} / \mathrm{s}$. Iso-surface with speed module equals to $0.3 \mathrm{~m} / \mathrm{s}$.

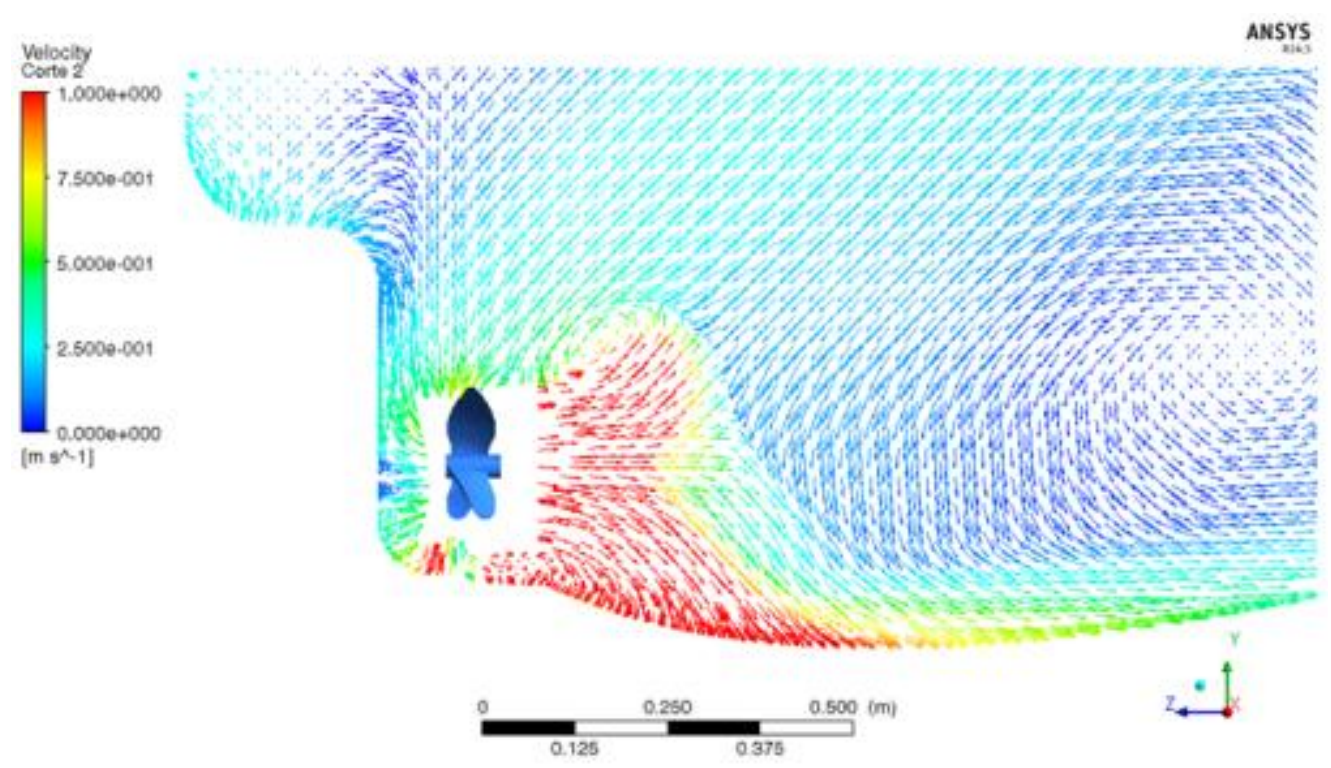

FIGURA 8. Representação vetorial dos perfis de velocidade devido à agitação mecânica. Vector representation of speed profiles according to mechanical stirring.

\section{Deposição de oxicloreto de cobre na parte inferior da parede do Reservatório}

\section{Resultados Experimentais}

Os resultados das coletas das amostras durante o esvaziamento do reservatório em agitação são apresentados na Tab. 2. É possível verificar que os valores dos desvios da concentração nos volumes retirados não ultrapassaram os limites normalizados $(+/-15 \%)$.

TABELA 2. Desvio em relação à concentração-base durante o esvaziamento do reservatório. Deviation of base concentration during tank emptying.

\begin{tabular}{llllllllll}
\hline Níveis de Amostragem & $4000 \mathrm{~L}$ & 3000L & 2000L & 1000L & 800L & $600 \mathrm{~L}$ & $400 \mathrm{~L}$ & 200L & Resíduo \\
\hline $\begin{array}{l}\text { Desvio em relação à } \\
\text { concentração base (\%) }\end{array}$ & $-5,21$ & $-7,5$ & $-9,2$ & $-4,43$ & $-4,88$ & $-1,97$ & $-0,38$ & 7,92 & 11,98 \\
\hline
\end{tabular}




\section{Análise da Tensão de Cisalhamento na parede do Reservatório}

A tensão de cisalhamento para o escoamento monofásico de um fluido co mo a água (fluido utilizado nesta simulação numérica) é determinada pela Lei de Newton da Viscosidade, em que a tensão é proporcional ao gradiente negativo da velocidade local. O fluido comunica parte da sua quantidade de movimento para a região imediatamente adjacente de líquido, na direção transversal ao escoamento. O fluxo da quantidade de movimento ocorre da região de maior velocidade para a região de menor velocidade. Assim, pode-se interpretar o gradiente de velocidade como sendo a força impulsora do transporte da quantidade de movimento (BIRD et al., 2002).

$$
\tau_{\mathrm{yx}}=-\mu \cdot \frac{d v_{x}}{d y}
$$

Essa variável de saída do modelo é importante para a análise da deposição da solução-teste na parede do reservatório, na região inferior do mesmo.

Da análise da [eq. (6)], é possível perceber que a variação da velocidade entre as diversas lâminas do escoamento promove regiões de maior ou menor tensão de cisalhamento. Quanto maior for o valor da tensão de cisalhamento na parede, significa que há um gradiente de velocidade maior nessa região, e, portanto, a movimentação do fluido é maior que em regiões de baixos valores de tensão. Com isso, regiões de baixos valores de tensão de cisalhamento na parede inferior do reservatório correspondem a regiões onde deverá ocorrer maior deposição de cobre.

Para garantir que os resultados próximos à parede sejam válidos, é necessário avaliar os valores do adimensional Yplus (distância adimensional com referência à parede, utilizado como informação sobre a resolução da malha próxima à mesma) em toda a parede do reservatório. Através dos estudos desenvolvidos por projetistas que utilizam os códigos de CFD, o valor desse adimensional varia de acordo com o modelo de turbulência adotado. No caso do modelo k-epsilon, adotado no presente trabalho, admitem-se valores de Yplus menores que 300. Para os demais modelos, admitem-se valores menores que 2 (NASCIMENTO et al., 2014).

Pode-se observar, na Fig. 9, que os valores do Yplus ficaram abaixo do limite para o modelo de turbulência adotado. Os maiores valores desse adimensional ocorreram na região influenciada pelo jato de fluido produzido pelo agitador hidráulico, que foi montado próximo à parede na região plana inferior do reservatório. Quanto maior o valor da velocidade tangencial à parede, maior o valor do Yplus, para uma mesma distância da parede.

As Fig. 10 a 13 mostram a tensão de cisalhamento na parede do reservatório com o valor máximo da legenda igual a 0,3 Pa e os detalhes das regiões de deposição de cobre obtidos nos resultados experimentais. Esse valor máximo da tensão foi escolhido após um estudo de correlação dos resultados da simulação numérica e aqueles encontrados experimentalme nte.

A Fig.10 mostra a influência do agitador hidráulico na deposição de cobre na região plana na parte inferior da parede do reservatório. É possível observar que a região que não é diretamente afetada pelo agitador, apresenta alta deposição de cobre.

As Fig. 11 e 12 mostram as regiões do apoio do berço do reservatório na lateral esquerda e na lateral direita do mesmo, respectivamente. Ambas as regiões apresentam problemas de agitação e consequente deposição de cobre, o que é possível perceber, também, da análise da isossuperfície da Fig. 7.

A Fig. 13 mostra a região de sombra do agitador mecânico na parte frontal do reservatório. Mesmo com a proximidade do agitador mecânico, que possui a maior contribuição no sistema de agitação instalado, essa região também apresenta deposição de cobre devido à localização da sua geometria numa posição anterior à hélice. Esse fato é percebido, também, da análise vetorial da Fig. 8. 
Portanto, a análise das Fig. 10 a 13 indica que as regiões de baixos valores de tensão de cisalhamento (valores menores que $0,3 \mathrm{~Pa}$ ), na parte inferior da parede do reservatório, correspondem às regiões de deposição de cobre.

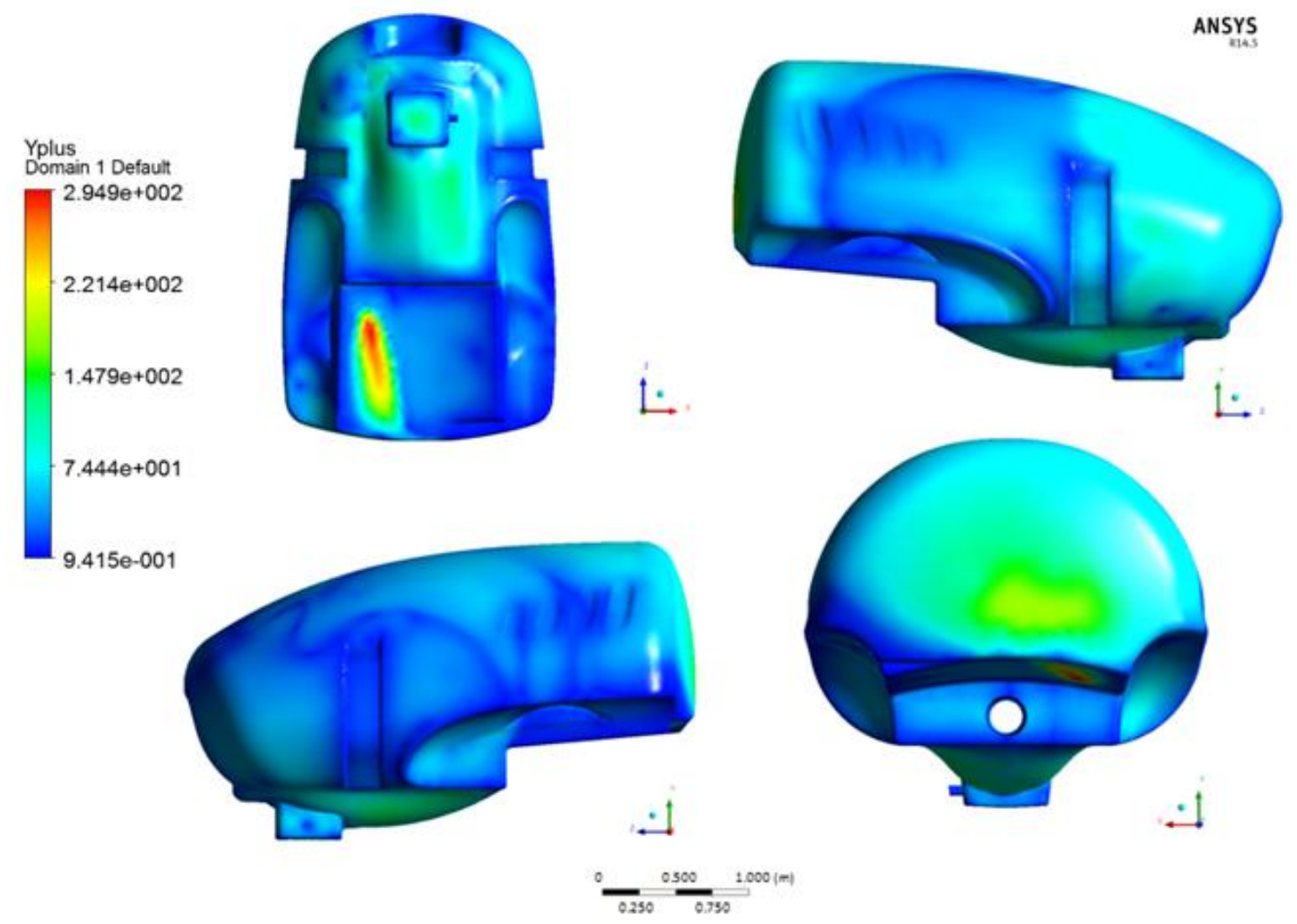

FIGURA 9. Valores do Yplus nas regiões da parede do reservatório. Yplus values on the tank wall regions.
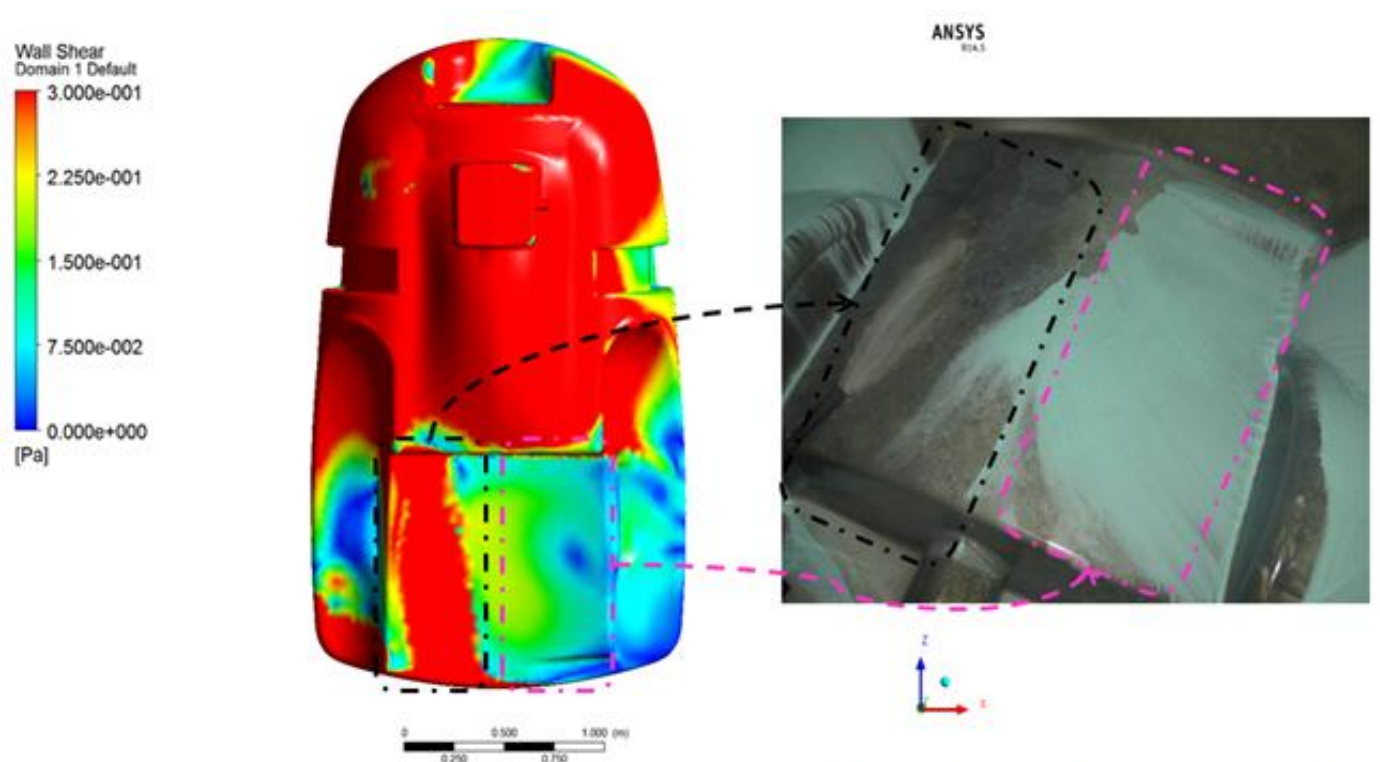

a) Limite superior da $\tau_{\text {parese }}$ igual a $0,3 \mathrm{~Pa}$.

b) Deposiçăo de cobre após esvaziamento do reservatório.

FIGURA 10. Comparação numérico-experimental entre os perfis de tensão de cisalhamento e as regiões de deposição de cobre na parte plana da parede inferior do reservatório. Numerical and experimental comparison between wall shear stress profiles and copper residue deposition regions on the flat portion of the tank bottom. 


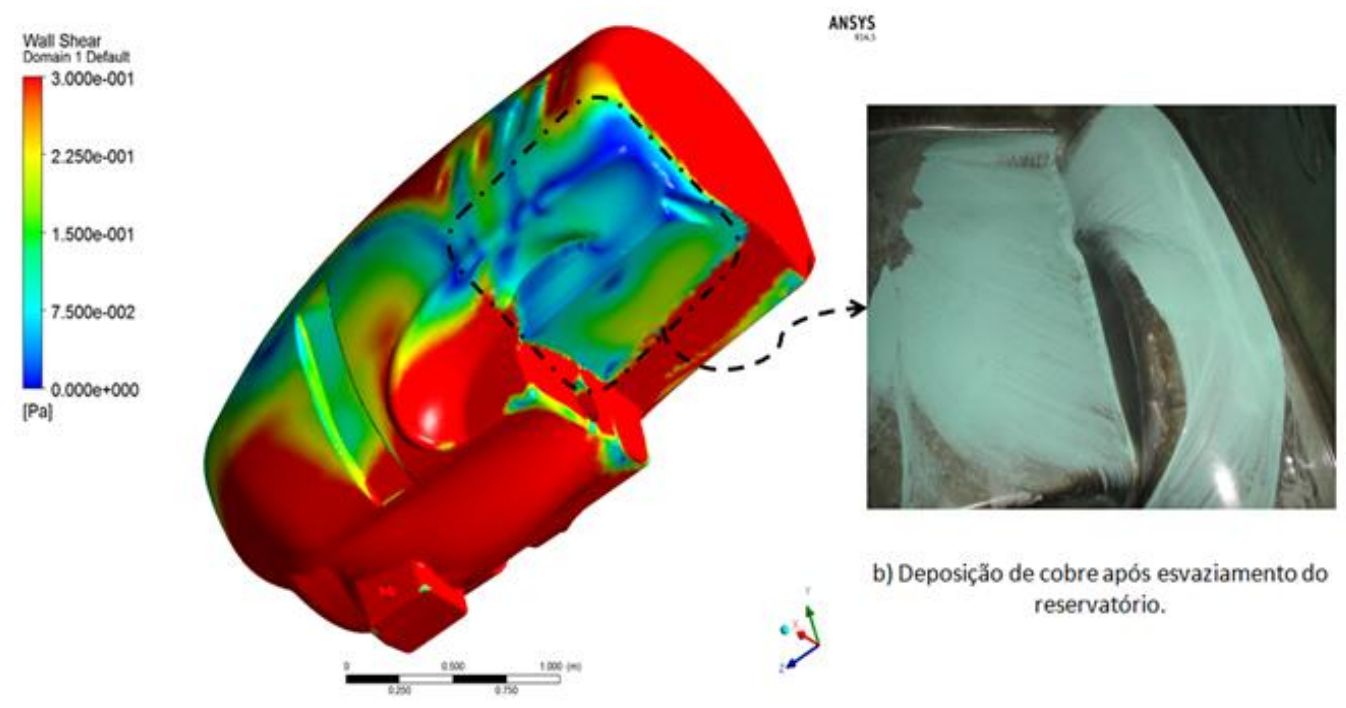

a) Limite superior da $\tau_{\text {parese }}$ igual a $0,3 \mathrm{~Pa}$.

FIGURA 11. Comparação numérico-experimental entre os perfis de tensão de cisalhamento e as regiões de deposição de cobre no apoio lateral esquerdo do berço do reservatório. Numerical and experimental comparison between wall shear stress profiles and copper deposition regions on the left side of tank support.

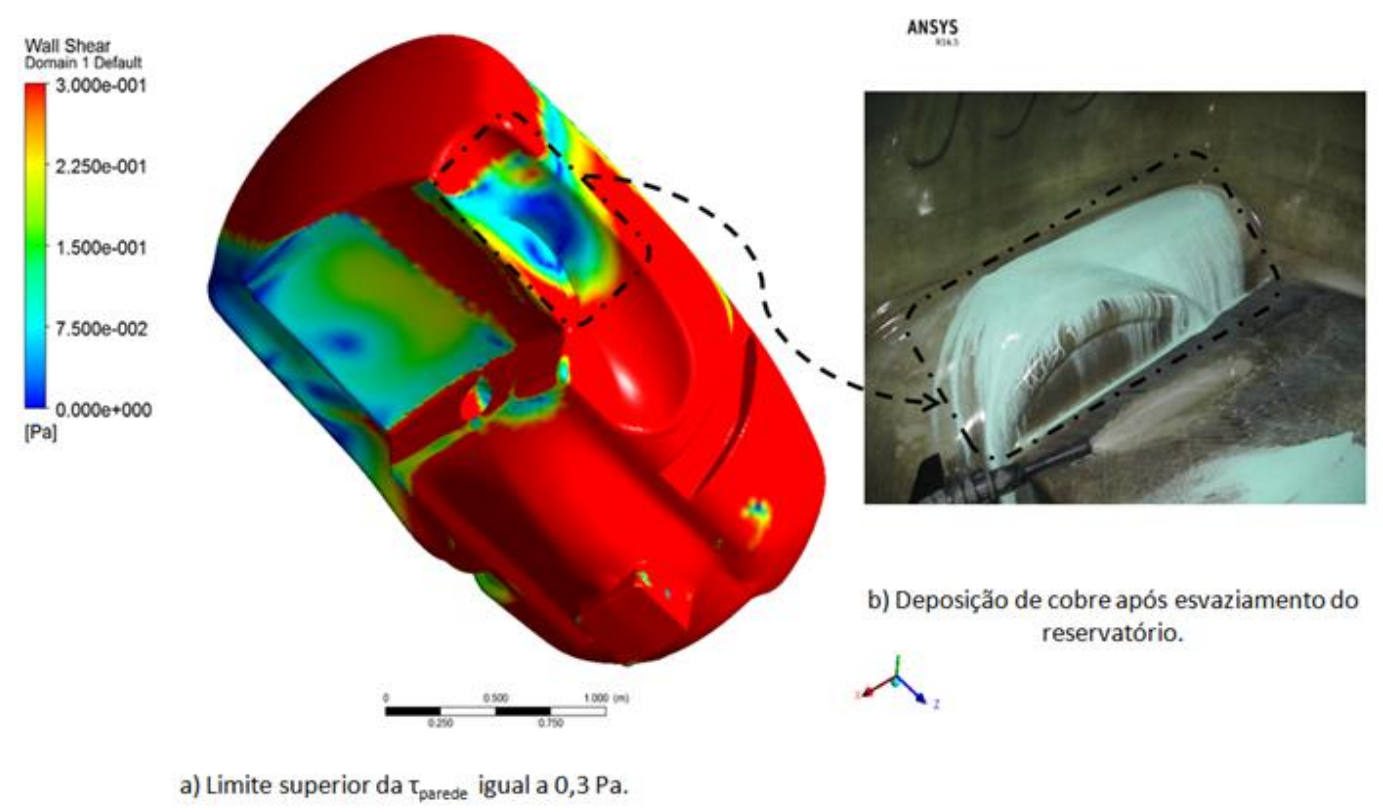

FIGURA 12. Comparação numérico-experimental entre os perfis de tensão de cisalhamento e as regiões de deposição de cobre no apoio lateral direito do berço do reservatório. Numerical and experimental comparison between wall shear stress profiles and copper deposition regions on the right side of tank support. 


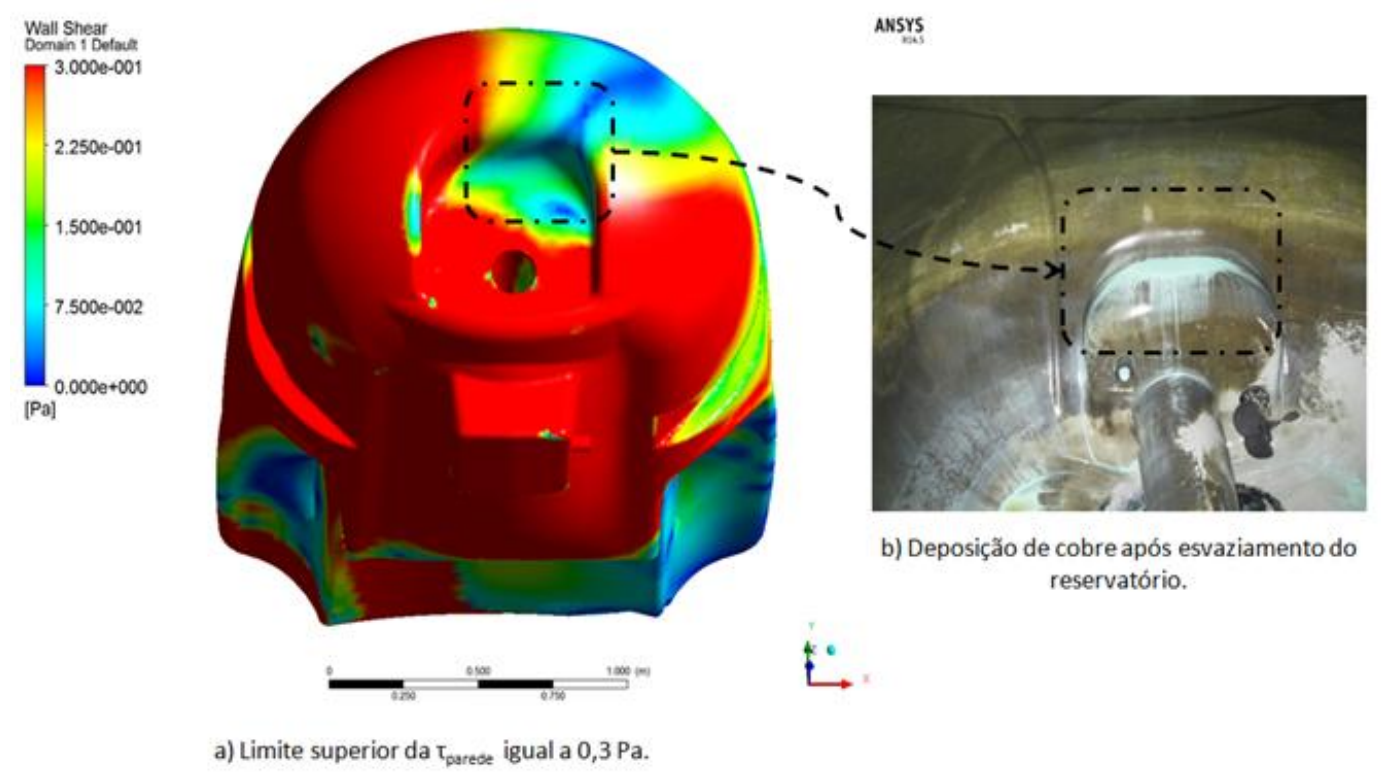

FIGURA 13. Comparação numérico-experimental entre os perfis de tensão de cisalhamento e as regiões de deposição de cobre na parte frontal do reservatório (região de sombra do agitador mecânico). Nume rical and experimental comparison between wall shear stress profiles and copper deposition regions on the tank front wall.

\section{CONCLUSÕES}

Através dos resultados obtidos nos testes experimentais e na simulação numérica utilizando um modelo monofásico do reservatório de 4.000 litros, foi possível concluir que:

- Dos resultados experimentais da concentração de oxiclore to de cobre nos três níveis do reservatório (fundo, meio e topo), a variação da concentração em ambas as coletas (preliminar e após 16 horas em repouso) foi inferior ao critério normalizado. Porém, a variação entre as coletas para um mesmo nível do reservatório foi superior ao critério normalizado devido à deposição da solução-teste na parede inferior do mesmo.

- Dos resultados das coletas das amostras durante o es vaziamento do reservatório em agitação, os desvios em relação à concentração base ficaram dentro dos limites do critério normalizado;

- Da análise dos perfis de velocidade ao longo de todo o reservatório, a agitação da calda é mais influenciada pelo agitador mecânico que pelo agitador hidráulico, sendo que há regiões de sombra de agitação na região plana da parte inferior da parede do mesmo.

- Não foi possível identificar nenhuma tendência ou relação direta entre os valores encontrados na simulação numérica de velocidade média no escoamento e os valores de concentração de cobre obtidos experimentalmente no interior do reservatório.

- Da análise da tensão de cisalhamento na parede do reservatório, as regiões com baixos valores numéricos da tensão (valores menores que $0,3 \mathrm{~Pa}$ ) corresponderam às regiões de deposição de cobre, com concentração acima do critério normalizado. As regiões com valores maiores que $0,3 \mathrm{~Pa}$ corresponderam às regiões onde não ocorreu deposição;

A simulação através do modelo monofásico é viável para avaliar a deposição de cobre em reservatórios de pulverizadores agrícolas. Para a avaliação da concentração ao longo do volume do reservatório, sugere-se um estudo da simulação numérica com um modelo multifásico Euler-Euler, que é o método que permitirá a verificação do mapa de concentração, sem a necessidade de uma correlação indireta. 


\section{REFERÊNCIAS}

ANSYS. CFX14.5 - User guide. Canonsburg, 2012.

AUGUSTO, P. E. D.; PINHEIRO, T. F.; CRISTIANINI, M. Utilização da fluidodinâmica computacional (CFD) na avaliação de tratamentos térmicos de bebidas em garrafas. Brazilian Journal of Food Technology, Campinas, v.13, n.4, p.260-270, out./dez. 2010.

BIRD, R. B.; STEWART, W. E.; LIGHTFOOT, E. N. Transport phenomena. 2 nd. ed. New Jersey: John Wiley \& Sons, 2002. p.12-13.

ENTAM - EUROPEAN NETWORKING FOR TESTING OF AGRICULTURAL MACHINES. prEN 12761. Sprayers and liquid fertilizer distributors - environmental protection - part 2: low crop sprayers and part 3: air-assisted sprayers for bushes and tree crops. Brussels, 1997. 17p.

ISO - INTERNATIONAL STANDARDIZATION ORGANIZATION. ISO 5682-2. Equipment for crop protection - spraying equipment - part 2: test methods for hydraulic sprayers. Switzerland, 1997.

NASCIMENTO, C. A. R.; LOPES, M. B.; MARIANI, V. C. Estudo numérico do escoamento turbulento de ar em tubos com ranhuras helicoidais. In: CONGRESSO NACIONAL DE ENGENHARIA MECÂNICA - CONEM, 8., 2014, Uberlândia. Anais...

SPOGIS, N. Metodologia para determinação de curvas de potência e fluxos característicos para impelidores axiais, radiais e tangenciais utilizando a fluidodinâmica computacional. 2002. 180f. Dissertação (Mestrado em Engenharia Química) - Universidade Estadual de Campinas, Campinas, 2002.

TAMBURINI, A.; CIPOLLINA, A.; MICALE, G.; BRUCATO, A.; CIOFALO, M. CFD simulations of dense solid-liquid suspensions in baffled stirred tanks: Prediction of suspension curves. Che mical Engineering Journal, New York, n.178, p.324-341, 2011.

UCAR, T.; FOX, R. D.; OZKAN, H. E.; BRAZEE, R. D. Simulation of jet agitation in sprayer tanks: Comparison of predicted and measured water velocities. Transactions of the ASAE, St. Joseph, v.44, n.2, p.223-230, 2001.

UCAR, T.; FOX, R. D.; OZKAN, H. E.; BRAZEE, R. D.; DERKSEN, R. C. Experimental study of jet agitation effects on agrochemical mixing in sprayer tanks. Journal of Agricultural

Engineering Research, London, v.75, p.195-207, 2000. 